\title{
Characterizing Interactions among Members of Deaf Communities in Orkut
}

\author{
Glívia A.R. Barbosa ${ }^{1}$, Ismael S. Silva ${ }^{1}$, Glauber Gonçalves ${ }^{1}$, Raquel O. Prates ${ }^{1}$, \\ Fabrício Benevenuto $^{2}$, and Virgílio Almeida ${ }^{1}$ \\ ${ }^{1}$ Computer Science Department, Federal University of Minas Gerais, Brazil \\ ${ }^{2}$ Computer Science Department, Federal University of Ouro Preto, Brazil \\ \{gliviaangelica, ismael.silva, ggoncalves, rprates, \\ fabricio, virgilio\} edcc.ufmg.br
}

\begin{abstract}
This paper presents a characterization of interaction phenomena among members of communities aimed at deaf people in an online social network system, namely Orkut. The results revealed that members of deaf communities are tighter than members of other communities. However, analysis of the interface indicates that it does not always address their specific needs.
\end{abstract}

Keywords: Social Networks, Online Communities, Deaf, Orkut, Accessibility.

\section{Introduction}

The challenges for deaf people to access or interact with social networks are often taken for granted, since most social networks do not involve audio elements in their interfaces. However, it is important to notice that the first language for deaf people is sign language and communication takes place, most of the time, in face-to-face situations [14]. Thus, having to interact through the written form of oral languages may be a challenge for deaf users [4], [10], [14]. Furthermore, deaf culture has many specific features that may impact how deaf users interact through social network systems.

One known feature of deaf culture is that interaction with other deaf people is extremely important and communities tend to be tighter [14]. Therefore, online communities can represent a space of autonomy, expression, communication and expansion of their relationship networks [15]. Thus, motivated by the importance that offline communities represent in the lives of deaf users and the benefits that online communities can offer them, the goal of this paper is to investigate and present the characterization of their friendship network, as well as the interaction phenomena among members of Orkut communities aimed at Brazilian's deaf users.

Orkut was chosen because it is the most popular social network system in the country [6]. In Orkut, a community refers to a virtual space to connect people who seek to discuss issues, express beliefs, values and share ideas freely [17]. Members of a community do not necessarily establish a friendship relationship among themselves [16]. The community can be defined as public or moderated and its members may interact through forums, polls and events.

The results revealed that the friendship network formed by community members aimed at deafs in Brazil is more connected than the network of users in communities 
with different focuses. However, when analyzing the interaction among them, mediated by available resources in the communities (e.g. forums, polls and events), the results indicate that communication among the members of deaf communities have often not been performed through these resources. The analysis identifies aspects of the proposed interaction which are challenging for deaf users and that may lead them not to use the available communication mechanisms.

This work contributes to the understanding of how deaf users are interacting through network systems, like Orkut. This understanding is important to the design of network systems which can be more accessible and usable by deaf users, allowing social network systems to become more inclusive.

\section{Related Work}

Currently there is a large number of works which focus on analyzing and characterizing phenomena in social networks (e.g. [1], [2], [3], [16] and [19]). Also there are some works regarding how deaf people use Information and Communication Technologies (ICTs) (e.g. [10], [12] and [15]) However, to the the best of our knowledge, an investigation aimed at characterizing interaction among members of deaf communities on social networks has not yet been published.

Papers that focus on analysis and characterization of social networks usually study their topology and/or interaction. In [1] and [16] the authors conducted a study in order to understand the structural characteristics that influence the evolution of online communities and social networks over time, whereas works like [2], [3] and [19] analyzed the user interaction in social networks in order to characterize it and provide relevant information for the development of future applications.

In papers that address the deaf and ICTs, the focus usually is on how ICTs can support communication of deaf users among themselves and with listeners. Works like [10] and [12] found that, as listeners, the deaf also want to communicate with a variety of people and quickly, but what differs is the language used in this interaction. Authors in [15] describe the important role of ICTs in the autonomy and socialization of the deaf, and note that not all the specific characteristics of these users have been considered in the interaction models of these systems.

None of the papers we have found analyze the use and interaction of deaf users through social network communities. In this work we present an analysis of the deaf culture in the use of communities in a social network system. Our goal is to characterize the interaction among members of network systems' communities aimed at deaf users in Brazil. The scope was limited to Brazilian's deaf users, since other works (e.g. [5]) have shown that culture has an impact on how people use social networks. Since in Brazil Orkut is the most popular social network, the investigation was done in this system.

\section{Methodology and Data Collection}

The methodology adopted to conduct this study consists of five steps: (1) definition of communities of interest, (2) data collection, (3) friendship network analysis, (4) use and interaction analysis and (5) characterization. 
Orkut does not allow its members to identify themselves as deafs, even if they wanted to. Thus, in order to analyze the deaf friendship networks we investigated members of communities aimed at deaf users. Communities that explicitly declared themselves as aimed at deaf people through their names or description were considered for the analysis. Examples of the selected communities names are: "Orkut Deafs in Brazil", "Yes, I am deaf', and "Deafs of Brazil Unite". We selected 14 deaf communities. In these communities $12 \%$ (average per community) of their members spontaneously declared to be deaf in the "name" field", in their profiles page (range $10-14 \%$ with $95 \%$ confidence).

For comparison we also selected other communities that focused on different subjects. We selected 6 communities aimed at homosexual users, 6 communities that connect people who have the same surname and 9 random communities. We collected in total data from 35 communities classified into 4 categories. Each community had between 100 and 500 members and all more than 4 years of existence. The data was collected from October $16^{\text {th }}$ to November $20^{\text {th }}, 2010$.

The chosen communities have between 100 to 500 members due to two main reasons. First, the fact that Orkut does not display the profile of all members if the community has more than 1000 members. Second, in order to compare the different communities they should have a similar range of members. Thus, this range allowed for a more accurate analysis of the desired communities.

For the friendship network analysis we collected the information of the profile and friendship network of 10.957 members, distributed in 35 communities described above. With this data, we verified the number of friends that each member contains in Orkut and how many of these friends are also in the community. This analysis allowed us to identify aspects related to intensity of relationships (i.e. connection) among community members.

The use and interaction analysis in communities was performed in order to complement the results obtained from the friendship network analysis. This analysis intended to identify what was the impact of this connection on the interaction among community members within the community itself. The method used in this step was the Semiotic Inspection Method (SIM) [8], grounded on the Semiotic Engineering Theory [7]. The choice of this method was due to its ability to provide a theoretical based analysis of the observed user-interface-user interaction as meant by the designers, as well as the actual interaction that took place among users of a community [8].

In the next section we present the characterization of the interaction among members of the deaf communities, based on the analysis of their friendship networks, as well as their interaction through the communities.

\section{Characterization}

Group interaction is extremely relevant to deaf people [14] and their communities tend to have tight relationships. Thus, our hypothesis is that online communities

\footnotetext{
1 Anytime the word "deaf" appeared in the "name" field the user was considered to be deaf. The information in the "About me" field was not considered since it would require a semantic analysis of the text.
} 
composed by deaf users also tend to have tighter clusters than other communities. Therefore, in our investigation we intended to answer the following questions: (1) Do deaf users have a closer relationship in online communities than other users? (2) If so, how does it impact their communication through Orkut communities?

\subsection{Characterization of the Friendship Network}

The following graphics show the distribution of friends of the community members in Orkut, in their own communities, and the relation between these values. Each curve represents the Cumulative Distribution Function (CDF) [13] of an analyzed community: the $\mathrm{X}$ axis represents the number of friends of community members; the $\mathrm{Y}$ axis represents the cumulative fraction of members in each community with $\mathrm{X}$ friends. For instance, the point $\mathrm{X}=200$ and $\mathrm{Y}=0.8$ in a curve of the community " $\mathrm{C}$ " means that $80 \%$ of the members of "C" have at most 200 friends and that $20 \%$ of the members of " $C$ " have more than 200 friends. The slower the CDF curves grow to reach 1 in the $\mathrm{Y}$ axis, the greater the number of friends of the community members.

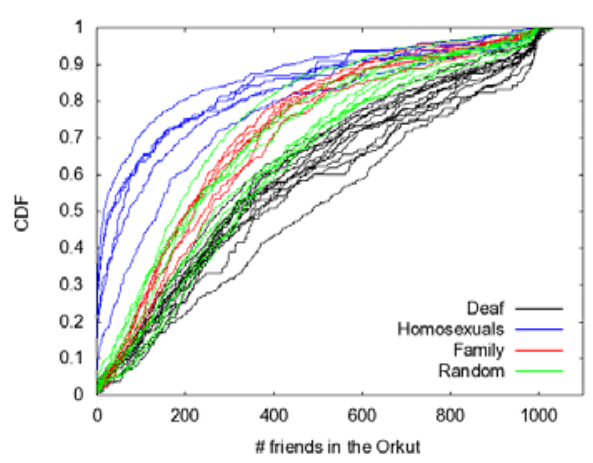

(a) CDF of the total number of friends of the members of each community

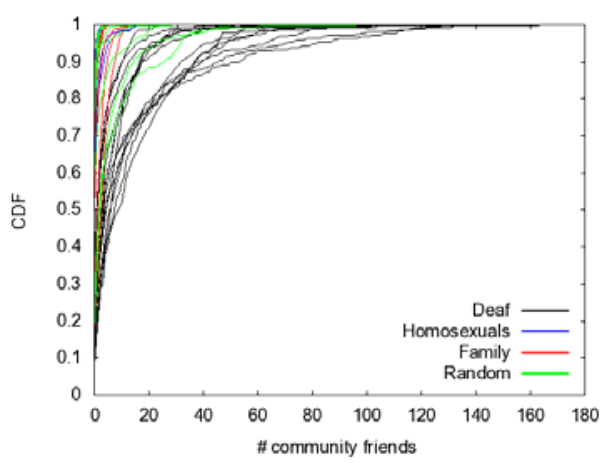

(b) CDF of number of friends within a community

Fig. 1. Number of friends that the analyzed Orkut community members have

The graphic depicted in the Fig. 1(a) presents the CDF of the number of friends that the community members have in Orkut and the graphic in Fig. 1(b) shows the $\mathrm{CDF}$ of the number of their friends who also participate in this community. We can note that among the 4 types of analyzed communities (i.e., deaf, homosexual, family and random selected), the members of communities related to the deaf have a higher number of friends in Orkut (see Fig. 1 (a)) and within their own communities (see Fig. 1 (b)). By comparing them, it is possible to see that members of deaf communities have more friends than members of the others (curves grow more slowly).

These results suggest that members of the analyzed deaf communities can be using Orkut to increase their friendship network. If they are also making new friends who are listeners, Orkut may be supporting the communication among deaf and listeners. This is an important finding, since face-to-face communication between deafs and listerners face the barrier of the different languages used [14], [10]. 
Next, we investigated the relation between the number of friends in the community and the number of friends in Orkut that each member of the analyzed communities has. These results are illustrated in Fig. 2 (a). To facilitate the visualization Fig. 2(b) shows only the most representative curves (i.e. median CDFs) of each analyzed group of community.

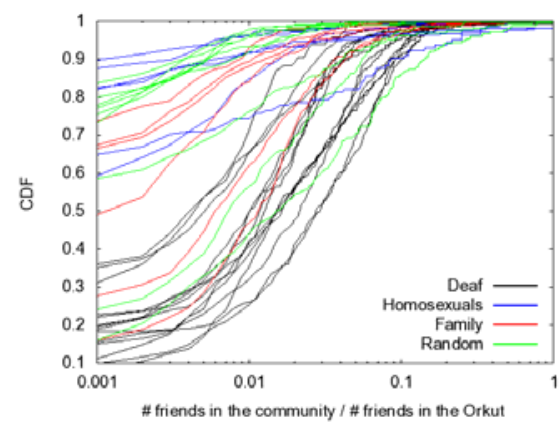

(a) $\mathrm{CDF}$ of rate of friends within the community that members of each analyzed community have

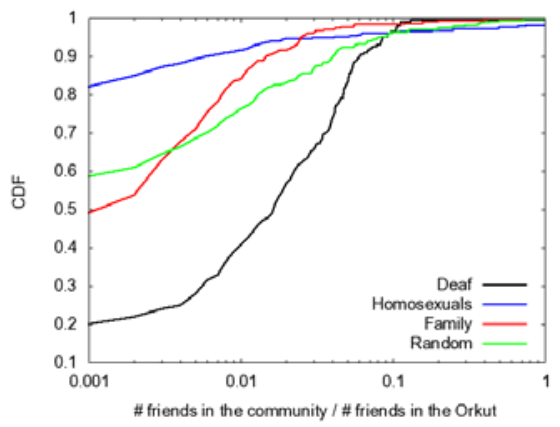

(b) Most representative curves of each group of community

Fig. 2. Relation between number of friends in community and total number of friends

Overall, we can note that members of deaf communities have a higher rate of friends within the community than the members of the other communities, since the deaf's curves grow more slowly than other ones. For instance, while $60 \%$ of members of the most representative deaf communities have more than $1 \%$ of their friends participating in the same community, only $25 \%$ of random community members, $15 \%$ of family community members and $8 \%$ of homosexual community members have the same proportion of friends within the community. If we look at other points of this graphic Fig. 2(b), the observed results will show that deaf users have a higher rate of friends who also participate in the same communities.

These results represent an evidence of a phenomenon among members of deaf communities called homophily by selection [9]. The establishment of relations motivated by selection indicates that people establish a relationship because they have "immutable" similar characteristics (e.g., disability or ethnicity) [9].

This observed phenomenon (i.e, homophily by selection) could be a reflection of offline behavior of the deaf community. According to [14] it is in the contact with their peers that deafs identify themselves and find stories similar to their own. The search for this deaf identity motivates the emergence of communities and associations which discuss the right to life, culture, education, work and welfare of all deaf [14].

The next section shows how this tighter relation among users of deaf communities impacts their use of the system.

\subsection{Characterization of the Use and Interaction}

The tighter relationship among deaf community members in Orkut raised the issue of how it impacted their interaction, that is, whether they used the available 
communication mechanisms differently or more often than other users. To investigate this issue the Semiotic Inspection Method [8] was used to analyze the possibilities made available by the system to its users; and how users actually use what is offered.

The analysis of what Orkut communities offered to users showed that there are 3 mechanisms - forums, polls and events - made available to whomever creates the community to decide which ones (if any) will be used by the community. All 3 mechanisms allow only for textual communication (no images or videos can be used). In communicating within a community, a member can only send messages to the whole community, no private messages can be sent. In order to check for updates in the ongoing communication, members must enter the community and access the thread of interest (users cannot request to be informed of updates).

These 3 aspects (text only, public messages and no informs) can be potential problems to all users. However, limiting communication to text can be a challenge for deaf users since their first language is usually sign language [4], [10], [14], which is visual and not textual. Thus, for deaf users there is an overhead of communicating in a language which is not their first language.

The analysis of the 35 communities considered showed that there were no relevant differences on the use of the communication mechanisms by deaf communities or the other ones. All the communities made available the 3 communication mechanisms (i.e., forums, polls and events) to its members (for creation and posting), but none of them had polls that had been opened in the year of 2010 or events listed for that year. Thus, the only mechanism actually used by community members was the forum. Looking specifically at the use of forums, in all communities new topics had been created in their forums in the previous month ${ }^{2}$. Nonetheless, the number of topics and posts were low $^{3}$, especially considering the number of members in these communities.

The analysis showed that although members of deaf communities have a tighter friendship network, this closeness does not impact the way they interact through the communities. In order to understand whether one of the reasons for this was the limitation to only communicate through text, we analyzed how members of the deaf communities who spontaneously declared themselves as deafs communicated in their personal scraps page (an environment outside the community).

Through this inspection it was possible to notice that deaf users receive many scraps $^{4}$ from their friends and the messages usually included videos, images or text. Most of them contained animations and short messages. The scraps that were mainly textual tended to be short (at most 2 sentences, each containing about 5 words). Another aspect that came to our attention was that often spam content received was not deleted from their profile. These spam messages were usually long texts, and it is probable that deaf users could not make sense of them and chose to leave them there.

Our analysis allows us to raise two possible explanations for the fact that members of deaf communities have closer relationships in the friendship network. The first is

2 Analysis was performed from $18^{\text {th }}$ to $25^{\text {th }}$ of November, 2010.

${ }^{3}$ In the 2 months previous to the analysis, only 4 communities which had new topics added to the forums in that period received more than 10 posts relative to the new topics.

${ }^{4}$ An Orkut functionality that allows one user to send a message to a friend in which different media can be used. 
that it is really a cultural trace of deaf communities, in which members tend to associate themselves to people who are similar to them (as suggested by our analysis of the networks). The inspection of the interface allows us to raise another hypothesis which is that due to the limiting communicative means in the communities, when members meet each other, they also include these new people as their personal friends in order to communicate with them more efficiently. Determining what role each of these possibilities in fact plays in the phenomenon of deaf communities being tighter than the others must be further investigated.

\section{Final Remarks and Future Work}

In this paper we investigated, analyzed and presented a characterization of the interaction among members of 14 Orkut communities aimed at connecting Brazilian's deaf users. The research of related literature led us to pose two questions that were investigated: (1) Do deaf users have a closer relationship in online communities than other users? (2) If so, how does it impact their communication through Orkut communities?

The answer to the $1^{\text {st }}$ question was obtained by the analysis of the deaf communities' friendship network, which showed that members of these communities are more closely connected than those of other communities with different focuses. To answer the $2^{\text {nd }}$ question we inspected Orkut community interface, as well as how members of deaf communities used them. This analysis showed that no relevant differences could be noticed between the interaction of members of deaf communities and those of the other communities considered.

The analyses allowed us to raise two possible explanations to the phenomenon of deaf communities having a closer connection: (1) it is a cultural trace of deaf communities; (2) it is caused or fostered by interface decisions that limit possibilities of communication through community mechanisms. The role played by these possibilities has to be further investigated. However, the identification of the closeness of the deaf communities, as well as the new raised questions contribute to HCI research since it characterizes relevant aspects of the interaction Orkut community members aimed at deaf users in Brazil. Moreover, the methodology adopted can contribute to researchers interested in investigating the use of network systems by users with deficiencies or other special needs.

The next steps in this research include interviewing deaf users of Orkut communities; investigating how quantitative analysis along time can help us better understand if there is a specific relationship pattern associated to this deaf members' closeness; and analyzing deaf users' relationships in other network systems to identify what observed aspects are culturally determined, as opposed to those that are a consequence of the interaction offered by the system.

Acknowledgments. This work is partially supported by the the INCT-Web (INWeb) (MCT/CNPq/ grant 57.3871/2008-6), and by the authors' individual grants and scholarships from FAPEMIG, CNPq, CAPES and UOL. 


\section{References}

1. Backstrom, L., Huttenlocher, D., Kleinberg, J., Lan, X.: Group formation in large social networks: membership, growth, and evolution. In: Proc. of ACM SIGKDD (KDD 2006), pp. 44-54. ACM, New York (2006)

2. Benevenuto, F., Rodrigues, T., Cha, M., Almeida, V.: Characterizing user behavior in online social networks. In: Proc. of ACM SIGCOMM. IMC 2009, pp. 49-62. ACM, New York (2009)

3. Burke, M., Marlow, C., Lento, T.: Feed me: motivating newcomer contribution in social network sites. In: Proc. of CHI 2009, pp. 945-954 (2009)

4. Cavender, A.C., Otero, D.S., Bigham, J.P., Ladner, R.: Asl-stem forum: enabling sign language to grow through online collaboration. In: Proc. of CHI 2010, pp. 2075-2078. ACM, New York (2010)

5. Chapman, C.N., Lahav, M.: International ethnographic observation of social networking sites. In: Proc. of CHI 2008 Extended Abstracts, pp. 3123-3128. ACM, New York (2008)

6. comSCORE.: Orkut Continues to Lead Brazil's Social Networking Market, Facebook Audience Grows Fivefold (2010), http: / / goo.gl / amro0

7. de Souza, C.S.: The semiotic engineering of HCI. MIT Press, Cambridge (2005)

8. de Souza, C.S., Leitão, C.F., Prates, R.O., Bim, S.A., da Silva, E.J.: Can inspection methods generate valid new knowledge in HCI? The case of semiotic inspection. International Journal of Human-Computer Studies 68(1-2), 22-40 (2010)

9. Easley, D., Kleinberg, J.: Networks, crowds, and markets: reasoning about a highly connected world. Cambridge University Press, Cambridge (2010)

10. Fajardo, I., Abascal, J., Cañas, J.J.: Bridging the digital divide for deaf signer users. In: Proc. of ECCE 2008, vol. 369, pp. 1-6. ACM, New York (2008)

11. Foucault Welles, B., Van Devender, A., Contractor, N.: Is a friend a friend?: investigating the structure of friendship networks in virtual worlds. In: Proc of CHI 2010 Extended Abstracts, pp. 4027-4032. ACM, New York (2010)

12. Henderson-Summet, V., Grinter, R.E., Carroll, J., Starner, T.: Electronic communication: themes from a case study of the deaf community. In: Baranauskas, C., Abascal, J., Barbosa, S.D.J. (eds.) INTERACT 2007. LNCS, vol. 4662, pp. 347-360. Springer, Heidelberg (2007)

13. Jain, R.: The Art of Computer Systems Performance Analysis. Wiley Computer Publishing, John Wiley \& Sons, Inc. (1991)

14. Lane, H., Hoffmeister, R., Bahan, B.: A journey into the Deaf-World. Dawn Sign Press (1996)

15. Martins, S., Filgueiras, L.: Evaluating interaction models for communication of deaf users. In: Proc of IHC 2010, pp. 193-196. 4 pages. SBC (2010) (in Portuguese)

16. Mislove, A., Marcon, M., Gummadi, K.P., Druschel, P., Bhattacharjee, B.: Measurement and analysis of online social networks. In: Proc. of IMC 2007, pp. 29-42. ACM, New York (2007)

17. Orkut.: Help (2010), http: / /www.google.com/support/orkut/bin/topic . py?topic=29543

18. Preece, J.: Online Communities: Designing Usability, Supporting Sociability. John Wiley \& Sons, Chichester (2000)

19. Wilson, C., Boe, B., Sala, A., Puttaswamy, K.P.N., Zhao, B.Y.: User interactions in social networks and their implications. In: Proc. of EuroSys 2009, pp. 205-218. ACM, New York (2009) 